\title{
Significance of Anterior Guidance in Selection of Posterior Teeth
}

\author{
Khurshid $\mathrm{AM}^{1 *}$ and Garg N ${ }^{2}$ \\ ${ }^{1}$ Department of Prosthodontics, Jazan University, Saudi Arabia \\ ${ }^{2}$ Department of Prosthodontics, SVS University, India
}

*Corresponding author: Khurshid A Mattoo, Assistant Professor, Department of Prosthodontics, College of Dentistry, Jazan University, Saudi Arabia, E-mail: drkamattoo@rediffmail.com

\section{Case Report \\ Volume 2 Issue 4}

Received Date: September 25, 2017

Published Date: October 09, 2017

DOI: $10.23880 /$ oajds-16000148

\section{Abstract}

The role of anterior guidance on the type of occlusion and overall stomatognathic health has been well documented in the literature related to Fixed Prosthodontics. Significance of proper anterior guidance for selection of posterior tooth forms for a removable partial denture is an area less explored by researchers. A female patient was treated with a cast partial denture for her Kennedy class 3 modification 1 partially edentulous situation, but reported back with a complaint of inefficient mastication. The patient had proclined anterior teeth with a resultant increased overjet which over a period of time affected anterior guidance. This abnormal anterior guidance has a direct bearing on the cuspal inclination of posterior teeth. Ideal treatment versus non ideal treatment in such cases has been discussed.

Keywords: Occlusion; Anatomic teeth; Condylar guidance; Cuspal inclination

\section{Introduction}

Selection of teeth is perhaps a topic that has received major attention of researchers since the geometric [1] classification of facial form and profile. Every concept [25] mentioned in the literature has focused mainly on the selection of size, shape, shade and occlusal anatomy of the artificial teeth. On the other side, occlusal concepts have been better understood in terms of biological and mechanical principles which has increased the scope of applied sciences in the past few decades. The influence of factors such as condylar guidance and anterior guidance on the overall stomatognathic health is well documented [6-8].

Although, role of anterior guidance has been applied to temperomandibular dysfunction its relationship to the selection of teeth for partial dentures has not been reported. This article in the form of a case report describes a case wherein existing faulty anterior guidance affected the selection of posterior teeth for a removable partial denture in terms of cuspal anatomy. The solution to such problem in terms of occlusal principles has also been discussed.

\section{Case Report}

A female patient aged 38 years, reported to the post graduate section of the department of Prosthodontics with chief complaint of persistent ineffective mastication (especially tearing and grinding) even after wearing a cast partial denture. The patient had got the partial denture made by a postgraduate student in the same department and was satisfied with the fit and esthetics of the partial denture but found little difference in masticatory efficiency before and after wearing dentures. All related histories were noncontributory and insignificant to the chief complaint. The treatment modality undertaken by the previous student was same as that for a typical Kennedy class 3 modification 1 situation. The partial 
edentulous arch on the left side included replacement of three posterior missing teeth while the modification space on the right side had only one premolar missing. The cast partial designing included occlusal rests for both partial edentulous spaces with simple circlet clasps engaging the anterior and posterior abutments. All retentive arms were flexible enough to retain the cast partial denture against dislodging forces. Upon placement of the partial denture in the patient's mouth, there were no occlusal interferences from any of the metal components and all natural teeth were in contact when the patient would occlude (Figure 1a,b). The patient, however, demonstrated wear facets on both anterior and posterior natural teeth in both arches. Maxillary anterior teeth were proclined resulting in increased overjet between the anteriors. Cuspal prominence of canines on both sides was lost (Figure 1c) and when the patient would protrude, the anterior guidance was not able to disclude the posteriors for a range of 3 to $4 \mathrm{~mm}$. The role of canine in mutual protection occlusion was hampered due to the above two reasons. The artificial teeth that were placed on the partial denture were lacking regular anatomic tooth cuspal anatomy (Figure 1d), suggesting that they were altered at the time of insertion as they would have been interfering in centric occlusion. Although the fit of the cast partial denture was satisfactory, grinding of cuspal inclination had reduced its masticatory efficiency, thus verifying the claims of the patient to be true.

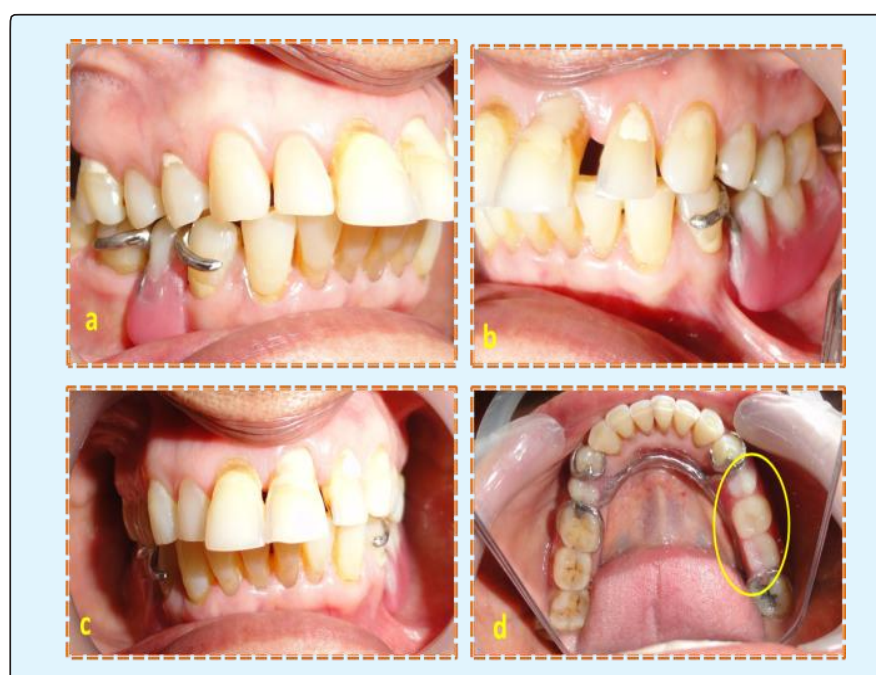

Figure 1: a,b: Cast partial denture with proper occlusion; c. increased overjet; d. reduced cuspal anatomy

The patient was educated about the problem by making diagnostic casts with a partial denture present, which were then mounted on a semi adjustable articulator that was programmed according to patient condylar guidance.
Both maxillary canines were then modified by wax up to demonstrate how cuspal inclines of posterior teeth are dependent on anterior teeth. The patient was advised for restoration in the form of either a complete or partial crown, of both canines with a steep anterior guidance to effect the change in posterior teeth. The option was declined by the patient.

\section{Discussion}

Anterior guidance that is favorable for optimal occlusion includes its ability to disclude the posterior teeth upon protrusion and lateral excursions. Unfavorable anterior guidance in natural dentition has deleterious effects that include anterior bone loss, tooth mobility 6 and more importantly the inability to limit the movement of the front end of the mandible [9-11]. In this case, maxillary and mandibular canines had worn out at the incisal surface, thus flattening them, which allowed the mandible to move anteriorly, rather than a downward direction. When the mandible is protruded or moved in a lateral excursion, the palatal slopes of the canine directs the mandible in a downward direction rather than moving forward. This feature is an important protective mechanism for the cusps even in natural teeth. The same mechanism explains the process of tooth wear in natural teeth once anterior guidance is lost or ineffective.

Treatment modality that was offered to the patient included restoration of either maxillary or mandibular canines with single crowns. Restoration of canines with new crowns having steeper palatal inclines would allow the mandible to assume its natural position when protruded or taken into a lateral excursion. This in turn would allow the artificial teeth on the removable partial denture to accommodate cusps with steeper inclines thus improving the masticatory efficiency. When the mandible is protruded, its movement is guided posteriorly by the condylar guidance of the mandible and anteriorly by the palatal inclines of the maxillary anterior teeth. When maxillary anterior teeth are proclined as in this case, the mandible has to travel some distance to bring palatal inclines of maxillary anterior teeth into effect. The distance travelled by the mandible under the sole influence of condylar guidance is horizontal with little or no vertical component. This in turn does not allow the use of steep cusps in the prosthesis. Ideally, treatment option in such cases includes orthodontic correction of proclined teeth, the extent of which is determined by the effective anterior guidance, esthetics and phonetics. Because orthodontic correction is time consuming and has age and financial limitations, most of the patients do not opt for such cumbersome procedure. Single modified crowns in 


\section{Open Access Journal of Dental Sciences}

relation to canines would have only produced effective anterior guidance while esthetic and phonetic objectives would have not been fulfilled.

\section{Conclusion}

Anterior guidance in its dynamicity defines and determines the cuspal form of posterior teeth and should be taken into consideration while selecting posterior teeth for a removable partial or a fixed partial denture.

\section{Acknowledgements}

The authors would like to clarify that there is no intention of criticism of the treatment done, but for the process of continued learning, it was necessary to point out at the lacunae.

\section{References}

1. Hasanreisoglu U, Berksun S, Aras K, Arslan I (2005) An analysis of maxillary anterior teeth: facial and dental proportions. J Prosthet Dent 94(6): 530-538.

2. Young HA (1954) Selecting the anterior tooth mould. J Prosthet dent 4(6): 748-760.

3. Pound E (1954) Lost fine arts in the fallacy of ridges. J Prosthet Dent 4(1): 6-16.
4. Frush JP, Fisher RD (1958) The dynesthetic interpretation of dentogenic concept. J Prosthet Den 8(4): 558-581.

5. Land LS (1996) Anterior tooth selection and guidelines complete denture esthetics. In: Winkler $\mathrm{S}$ (Ed.) Essentials of complete denture Prosthodontics. 2 St. Louis: Ishiyaku Euro America Inc pp: 200-216.

6. Schuyler H (2001) The function and importance of incisal guidance in oral rehabilitation. J Prosthet Dent 86(3): 219-232.

7. Schuyler CH (1953) Factors of occlusion applicable to restorative dentistry. J Prosthet Dent 3(6): 772-782.

8. Rosenstiel SF, Land MF, Fujimoto J (2001) Contemporary fixed prosthodontics. $3^{\text {rd }}$ (Edn.), Mosby pp: 202-213.

9. Ross IF (1974) Incisal guidance of natural teeth in adults. J Prosthet Dent 31(2): 155-162.

10. Broderson SP (1978) Anterior guidance: The key to successful occlusal treatment. J Prosthet Dent 39(4): 396-400.

11. Dawson DE Evaluation, diagnosis and treatment of occlusal problems. St. Louis: C.V. Mosby. 